\title{
Variations saisonnières du régime alimentaire du chevreuil (Capreolus capreolus) selon le sexe en milieu forestier à forte densité (forêt domaniale de Dourdan)
}

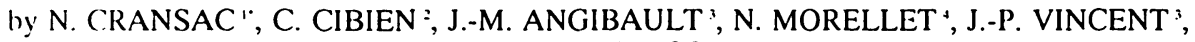 \\ et A.J.M. HEWISON * \\ 'Ecole Pratique des Hautes Etudes, Sciences de la Vie et de la Terre. \\ l.aboratoire de Biogéographie et Ecologie des Vertébrés, place Eugène-Bataillon, \\ F 34095 Montpellier Cedex 5 France. \\ 'Ecotone, BP 34, F 31321 Castanet-Tolosan Cedex, France.
}

'Institut de Recherche sur les Grands Mammifères, Institut national de la Recherche agronomique, BP 27, F 31326 Castanet-Tolosan Cedex, France.

e-mail: hewison@toulouse.inra.fr

'Institut de Recherche pour l'Ingénierie de l'Agriculture et de l'Environnement,

Unité de Recherche et d'Expertise Ecosystèmes Forestiers et Paysages, Groupement de Nogent-sur-Vernisson, Domaine des Barres,

F 45290 Nogent-Sur-Vernisson, France.

* Adresse actuelle: Arbre et Paysages d'Autan, Place de la Mairie,

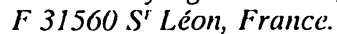

Summary. - Roe deer are selective but opportunistic feeders which are predicted to vary their diet in relation to scasonal variation in plant availability. Scasonal variation in diet is often difficult to assess because of a lack of samples outside the hunting season. Here we describe seasonal - and sex-related variations in forest roc deer diet estimated from the examination of rumen contents collected in each of the four seasons. Only six plant items comprised $80-90 \%$ dry weight of the rumen contents, with two, bramble and honcysuckle, virtually ubiquitous in the samples throughout the year. Otherwisc, the roc deer's diet varicd markedly between seasons, feeding on evergreen plants (such as heather and ivy) and some grasses in autumn and winter, but preferring herbs (anemonc) and the young leaves and buds of trecs (hawthorn, hornbeam, oak) in spring and summer. The roe deer was particularly opportunistic in exploiting the abundant, but cphemerally available, resources provided by fruits and nuts (e.g. blackberries in summer, acorns in autumn). There were sone differences between the sexes in the way that they exploited these seasonal resources, possibly duc to differences in energy requirements, but more likely due to differing behavioural patterns. Our results support the observation that the impact of roe deer on the vegetation in this forest mostly concerned bramble and honcysuckle.

Résumé. - Le chevreuil est un consommateur sélectif, mais opportuniste, qui adapte son régime alimentairc selon les variations saisonnières de la disponibilité des ressources. Les variations saisonnières du régime alimentairc sont souvent difficiles à mettre en évidence par l'examen du contenu des panses, en raison du manque d'échantillons en dehors de la période de chasse. Dans cet article, nous décrivons les variations, lićes à la saison et au sexe, du régime ali-

Mammalia, $t .65, n^{\circ} 1,2001: 1-12$. 
mentaire du chevreuil forestier estimées par cette méthode, sur des panses récoltécs à chacunc des quatre saisons. Six plantes représentaient à elles seules 80 à $90 \%$ du poids sec du contenu des panses, dont deux, la ronce et le chèvrefeuille, étaient présentes dans pratiquement chaque échantillon toute l'annće. Ceci mis à part, le régime alimentaire variait fortement entre les saisons. Le chevreuil consomme des végétaux à feuillage persistant (bruyère, lierre) et quelques graminées en automne et hiver, mais préfère les herbacées non graminéennes telles que l'anémone sylvie, et les bourgeons et jcunes feuilles d'arbres et arbustes (charme, chêne, aubépine) au printemps et en été. Le chevrcuil est particulièrement opportuniste en exploitant les ressources abondantes, mais éphémères, fournies par les fruits et les graines (les mûres en été, les glands en automne). Des différences entre les sexes sont apparues dans la façon d'exploiter les ressources saisonnières. Il est possible que des différences de besoins énergétiques en soient la cause, mais plus vraisemblablement des patrons comportementaux différents entre les sexes. Nos résultats confirment l'observation que l'impact du chevrcuil sur la végćtation de la forêt de Dourdan concerne principalement la ronce et le chèvrefeuille. nières.

KEY WORDS : C.capreolus, régime alimentaire, ronce, chèvrefeuille, variations saison-

\section{INTRODUCTION}

Depuis 30 ans, le chevreuil est en pleine expansion démographique et géographique en France (Boisaubert et Mouron 1997), comme partout ailleurs en Europe (Danilkin et Hewison 1996). Cette évolution pose de nombrcux problèmes pour la gestion de l'équilibre agro-sylvo-cynégétique (c.g. dégâts sylvicoles, Groupe chevreuil français 1999). C'est une petite espèce de Cervidés habitant généralement en forêt, pourvu d'un système social peu grégaire et d'un mode d'alimentation sélectif (Hewison et al. 1998). Il est considéré comme un cucillcur qui se nourrit préférentiellement de pousses, feuilles et fruits, en particulier celles des arbrisseaux, arbustes et arbres (Duncan et al. 1998). Il s'alimente aussi des jeunes herbacées non graminéennes, mais consomme peu de graminées. Son régime varie en fonction de la disponibilité de la végétation, c'est-à-dire de l'habitat et de la saison (Cornelis et al. 1999), et il doit être considéré aussi comme une espèce opportuniste (Tixier et Duncan 1996). En effet, sa flexibilité écologique et comportementale lui a permis de coloniser la plaine agricole depuis quelques décennies (Hewison et al. 1998) où il se nourrit d'espèces cultivées (Cibien et al. 1995 ; Duncan et al. 1998).

Il semble que le régime alimentaire du chevreuil varie selon les saisons (e.g. en fonction de l'abondance et de la périodicité de différentes espèces végétales (Danilkin et Hewison 1996), mais peu d'études ont pu démontrer cette variation pour une seule population dans un seul contexte environnemental. Ainsi, la littérature rapporte que le nombre d'espèces végétales consommées par le chevreuil est en général compris entre 3 et 42 (e.g. Cederlund et al. 1980), mais ce chiffre est souvent plus faible pendant l'hiver (Danilkin et Hewison 1996). De plus, selon les saisons, ses besoins doivent varier en fonction du niveau de son métabolisme et de son cycle de reproduction (Sempéré et al, 1998). Ainsi, on pourrait s'attendre à une différenciation de régime entre les sexes reflétant le cycle métabolique (Hewison et al. 1996) et les activités de reproduction : l'élevage des jeunes pour la chevrette (Andersen et al. 1998), la territorialité et le rut pour le brocard.

L'organisation et le fonctionnement d'une population de chevreuils forestiers ont été étudiés sur une période de 10 ans (1980-1990) dans une forêt du Bassin Parisien. 
L'occupation de l'espace et l'organisation sociale ont constitué les axes principaux de cette étude (voir Vincent et al. 1995). A l'occasion du tir d'animaux destiné à diminuer la densité de la population, il fut procédé à un prélèvement systématique du contenu des panses. L'objectif de ce prélèvement était d'établir le régime alimentaire des animaux sur un échantillon important afin d'apprécier d'éventuelles variations dues à la saison et au sexe.

\section{MATÉRIEL ET MÉTHODES}

Le site. - Ces études ont été réalisées dans la forêt domaniale de Dourdan $(40 \mathrm{~km}$ au sud-ouest de Paris $48^{\circ} 19^{\prime} \mathrm{N}, 02^{\circ} 01^{\prime} \mathrm{E}$, Fig. 1). C'est unc forêt ouverte d'environ 900 ha, constituée principalement de feuillus ( $90 \%$ de chênes rouvres et pédonculés, Quercus sessiliflora, $Q$. pedunculata ; $10 \%$ de pin sylvestre, Pinus sylvestris). Le sousétage est constitué en majorité de ronce (Rubus sp.), de charme (Carpinus betulus), d'aubépine (Crataegus monogyna), de chèvrefeuille (Lonicera peryclimenum) et d'un moindre degré de bouleau (Betula verrucosa), de troène (Ligustrum vulgare), de prunellier (Prumus spinosa), de lierre (Hedera helix) et de houx (Ilex aquifolium). Elle porte un peuplement de taillis sous futaie, traité depuis 1959, en conversion et futaie régulière de chênes et de pins sylvestres (Bideau et al. 1983; Ballon et al. 1992). La forêt est située en zone agricole renfermant quelques petits massifs boisés. Elle est bordée à l'ouest par l'autoroute A10 et le TGV.

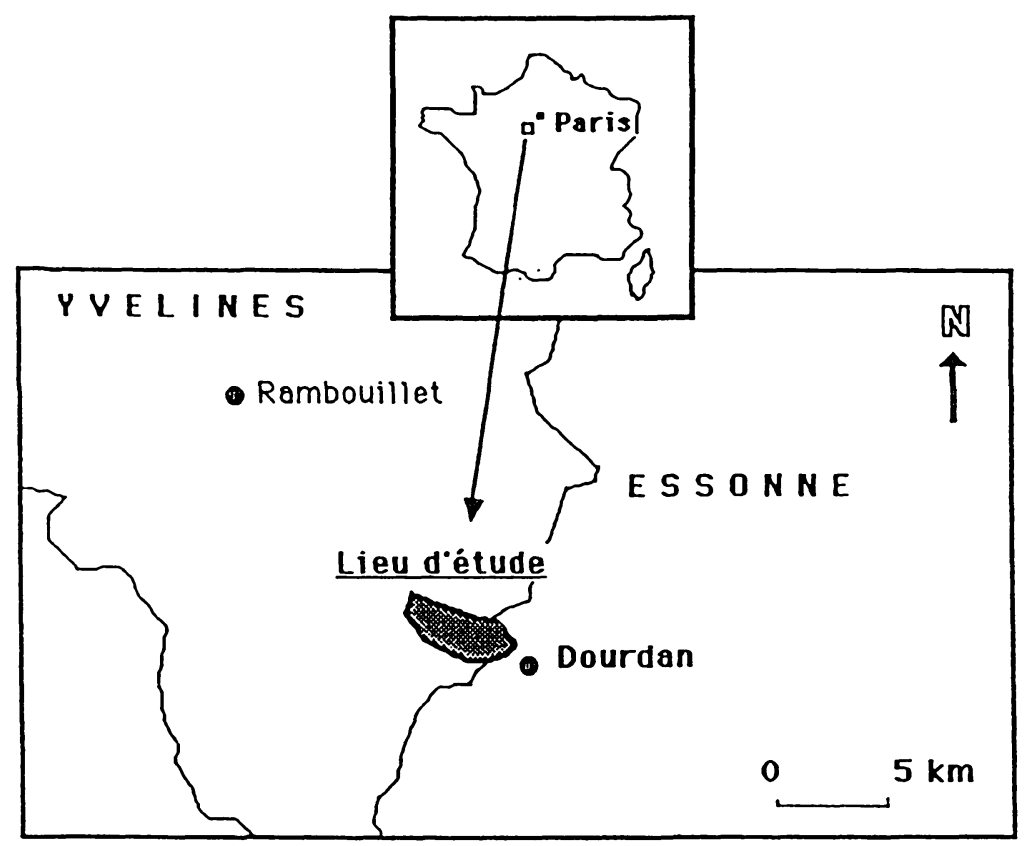

Fig. 1. - Localisation du site d'étude. 
La population de chevreuils. - Le chevreuil constituait l'espèce d'ongulés principale présente dans la forêt pendant l'étude, bien qu'un petit nombre de cerfs (Cervus elaphus) et de sangliers (Sus scrofa) y fissent des apparitions irrégulières. Jusqu'en 1979, la forêt était régulièrement chassée avec une récolte annuelle d'environ 10 chevreuils. Par la suite, et jusqu'à l'arrêt de l'étude, aucun prélèvement cynégétique n'cut lieu, ce qui permit à la population de se développer et de passer de 5 animaux /100 ha en 1980 à $25 / 100$ ha en 1988 (Vincent et al. 1991). Malgré son influence sur la disponibilité relative de certaines plantes (Ballon et al. 1992), cette augmentation des effectifs n'a pas globalement entraîné de modifications radicales de la composition floristique du site.

Le prélèvement. - En 1989 et 1990, 120 animaux au total ont été tirés (mâles, femelles, adultes et juvéniles de plus de trois mois). Les tirs furent répartis annuellement sclon quatre périodes ( 15 animaux par période; 60 par an) correspondant chacune à une saison (hiver : février ; printemps : avril ; été : août ; automne : novembre). Sur chaque animal tué, I'cnsemble du contenu de la panse était mélangé, et un échantillon de 750 grammes était prélevé, puis conservé au congélateur. Sur les 120 échantillons prélevés, 93 furent effectivement analysés (Tableau 1).

TABIEAU 1. - Nombre de panses analysées par sexe, saison et année de tir.

\begin{tabular}{cccccccccc}
\hline & \multicolumn{2}{c}{ Printemps } & \multicolumn{2}{c}{ Eté } & \multicolumn{2}{c}{ Automne } & \multicolumn{2}{c}{ Hiver } \\
\hline & Femelle & Mâle & Femelle & Mâle & Femelle & Mâle & Femelle & Mâle & Total \\
1989 & 1 & 5 & 3 & 4 & 6 & 9 & 8 & 7 & 43 \\
1990 & 6 & 9 & 4 & 7 & 9 & 6 & 2 & 7 & 50 \\
Total & 7 & 14 & 7 & 11 & 15 & 15 & 10 & 14 & 93 \\
\hline
\end{tabular}

Technique d'analyse des panses. - Les échantillons de contenus de panses étaient d'abord homogénéisés, puis 100 grammes étaient prélevés pour être ensuite lavés et filtrés sur des tamis de $5 \mathrm{~mm}$ et $2 \mathrm{~mm}$. Les refus des deux tamis ont été identitiés sous loupe binoculaire $(\times 25)$. Lorsque les caractères macroscopiques étaient insuffisants pour permettre la détermination des fragments, celle-ci a été réalisée sous microscope en s'aidant de la clé de détermination Plantox (Badia et al. 1986). Les fragments végétaux ainsi triés ont été ensuite séchés à l'étuve $\left(24\right.$ heures à $\left.60^{\circ} \mathrm{C}\right)$ et pesés au $1 / 1000 \mathrm{~g}$ (voir Maizeret et al. 1989).

Le traitement et I'analyse des données. - Pour élaborer le tableau de données, nous avons considéré les panses comme individus, caractérisés par 5 variables : la saison, le sexc de l'animal, l'espèce ou catégorie d'espèces (item alimentaire) concernée, sa présence/absence et son abondance (proportion du poids sec) dans l'échantillon. Les données des deux années ont été analysées ensemble. Nous n'avons pas considéré l'influence de l'âge sur le régime alimentaire car l'estimation d'âge à partir d'usure des dents est imprécise pour le chevreuil (Hewison et al. 1999). De plus, à partir de trois mois, le chevreuil trouve son régime d'adulte (Danilkin et Hewison 1996), l'âge minimum dans notre échantillon. L'analyse a testé l'influence du sexe de l'animal et de la 
saison de prélèvement sur la présence/absence (modèle log-linéaire) ou l'abondance (ANOVA sur des données transformées par arcsinus) des items consommés en considérant l'item comme facteur. Dans les deux cas, nous avons commencé par le modèle complet comprenant les 3 facteurs et leurs interactions. Nous avons ensuite utilisé une procédure par retraits successifs pour rechercher la signification des interactions puis celle des facteurs (Crawley 1993). Dans le cas d'un résultat significatif, nous avons procédé à une analyse par item pour identifier ceux qui variaient significativement entre sexe ou saison. Le logiciel SPSS fut utilisé pour ces analyses.

\section{RESULTATS}

Fréquence des diverses plantes dans les panses. - L'analyse des échantillons prélevés dans les panses nous a permis de distinguer 21 items alimentaires (voir Tableau 2).

L'analyse log-linéaire a porté sur un tableau de 1953 lignes (93 panses $\times 21$ items). Aucune interaction à trois facteurs n'apparaît $\left(\chi^{2}=44.4, \mathrm{dl}=60, \mathrm{p}=0.934\right)$. Il en cst de même pour les interactions item*sexe $\left(\chi^{2}=22.9, \mathrm{dl}=20, \mathrm{p}=0.294\right)$ et saison*sexe $\left(\chi^{2}=4.2, \mathrm{dl}=3, \mathrm{p}=0.241\right)$. Par contre, il existe une forte interaction significative saison*item $\left(\chi^{*}=291.3, \mathrm{dl}=60, \mathrm{p}<0.0001\right)$, c'est-à-dire que la variation de la fréquence saisonnière des items alimentaires est différente selon l'item considéré (Tableau 2).

En toutes saisons ronces et chèvrefeuille restent les deux items les plus largement représentés dans le régime des chevreuils ( $>70 \%$ des panses, $\mathrm{CV}=2$ et $12 \%$, Tableau 2). Pour les autres items, le coefficient de variation entre saisons est beaucoup plus important. et l'on note une sorte de dichotomie dans la représentation des items entre la période printemps-été et la période automne-hiver. Dans le premier cas, le charme $\left(\chi^{2}=48.6, \mathrm{dl}=3, \mathrm{p}<0.0001\right)$ et le chêne $\left(\chi^{2}=24.1, \mathrm{dl}=3, \mathrm{p}<0.0001\right)$ se retrouvent dans 44 à $76 \%$ des panses prélevées au printemps et en été, l'anémone $\left(\chi^{2}=22.2\right.$, $\mathrm{dl}=3, \mathrm{p}<0.0001)$ et l'aubépine $\left(\chi^{2}=19.4, \mathrm{dl}=3, \mathrm{p}<0.0001\right)$ sont fréquemment ingérées au printemps et les mûres $\left(\chi^{2}=34.4, \mathrm{dl}=3, \mathrm{p}<0.0001\right)$ sont particulièrement consommées en été. Dans le second cas, les espèces végétales pérennes, le lierre $\left(\chi^{2}=6.3\right.$, $\left.\mathrm{dl}=3, \mathrm{p}=0.097\right)$, les graminécs $\left(\chi^{2}=9.3, \mathrm{dl}=3, \mathrm{p}=0.025\right)$ et les bruyères $\left(\chi^{2}=17.5, \mathrm{dI}=3, \mathrm{p}<0.0001\right)$, ainsi que les champignons $\left(\chi^{2}=26.9, \mathrm{dl}=3\right.$, $\mathrm{p}<0.0001)$ et les glands $\left(\chi^{2}=59.5, \mathrm{dl}=3, \mathrm{p}<0.0001\right)$, apparaissent fréquemment dans le régime automnal et hivernal. Les autres items identifiés ne sont présents que de façon occasionnelle.

Pour tester la différence de fréquences des items dans les panses entre mâles et femelles, nous avons découpé le tableau de données et procédé à une analysc par saison (voir Tableau 1 pour la taille des échantillons). Aucune différence significative de la fréquence des items alimentaires entre panses des deux sexes n'apparaît au cours des saisons (printemps: $\chi^{2}=19.8, \mathrm{dl}=20, \mathrm{p}=0.470$; été : $\chi^{2}=9.9, \mathrm{dl}=20, \mathrm{p}=0.970$; automne $: \chi^{2}=25.4, \mathrm{dl}=20, \mathrm{p}=0.187$; hiver $\left.: \chi^{2}=12.2, \mathrm{dl}=20, \mathrm{p}=0.909\right)$.

Abondance des diverses plantes dans les panses. - L'analyse de variance sur le tableau de données montre une interaction triple significative $\left(F_{59,1952}=1.40, p=0.024\right)$, indiquant la complexité de la relation item-saison-sexe, nous imposant de traiter les données par sexe puis par saison. Pour les deux sexes nous avons trouvé des différences significatives d'abondance des plantes reconnues dans les panses selon la saison (mâles : $F_{59,1133}=6.32, p<0.0001$; femelles: $F_{59,818}=15.85, p<0.0001$ ). Ceci est notamment dû à une masse importante de glands $\left(F_{3,50}=11.60, p<0.0001\right)$ dans les panses en automne et, à un moindre degré, en hiver. De même, la ronce $\left(F_{3.50}=51.88\right.$, 
TABLEAU 2. - Fréquence des différents items alimentaires identifiés (proportion des panses dans lesquelles l'item est retrouvé) en fonction de la saison du prélèvement. Le coefficient de variation est calculé pour chaque item à partir des movennes saisonnières et donne un indice de la variabilité de la consommation de l'item entre saisons.

\begin{tabular}{lccccc}
\hline Item alimentaire & Printemps & Eté & Automne & Hiver & Coefficient \\
\hline Anémone sylvie (Anemone nemorosa) & 38.1 & 0.0 & 3.3 & 0.0 & 179.2 \\
Aubépine (Crataegus monogyna) & 33.3 & 5.6 & 0.0 & 0.0 & 164.1 \\
Bouleau (Betula verrucosa) & 4.8 & 0.0 & 3.3 & 0.0 & 119.0 \\
Bruyères (Calluna vulgaris, Erica & 4.8 & 0.0 & 36.7 & 29.2 & 102.0 \\
Champignons & 14.3 & 16.7 & 73.3 & 54.2 & 73.1 \\
Charme (Carpimus betulus) & 76.2 & 50 & 6.7 & 0.0 & 109.1 \\
Chênes (Quercus spp.) & 47.6 & 44.4 & 16.7 & 0.0 & 84.0 \\
Chèvrefeuille (Lonicera peryclimenum) & 71.4 & 94.4 & 90 & 83.3 & 11.8 \\
Germandrćc (Teucrium scorodomia) & 14.3 & 16.7 & 16.7 & 25.0 & 25.9 \\
Glands & 14.3 & 5.6 & 93.3 & 29.2 & 111.6 \\
Graminées & 14.3 & 16.7 & 43.3 & 45.8 & 56.2 \\
Houx (Ilex aquifolium) & 0.0 & 0.0 & 0.0 & 4.2 & 200.0 \\
Labiées & 0.0 & 0.0 & 3.3 & 0.0 & 200.0 \\
Lierre (Hedera helix) & 14.3 & 27.8 & 43.3 & 41.7 & 42.8 \\
Monocotylédones & 4.8 & 0.0 & 0.0 & 4.2 & 116.0 \\
Mousses & 14.3 & 5.6 & 26.7 & 16.7 & 55.0 \\
Mûre & 0.0 & 50.0 & 0.0 & 0.0 & 200.0 \\
Onagracées & 0.0 & 0.0 & 3.3 & 0.0 & 200.0 \\
Prunellier (Prumus spinosa) & 4.8 & 0.0 & 3.3 & 0.0 & 119.0 \\
Ronce (Rubus sp.) & 0.0 & 11.1 & 10.0 & 0.0 & 115.8 \\
Troène (Ligustrum vulgare) & 100.0 & 100.0 & 100.0 & 2.4 \\
\hline
\end{tabular}

$\mathrm{p}<0.0001)$ est très abondante dans les panses de chevreuils en hiver. A l'inverse, d'autres plantes, en l'occurrence le chèvrefeuille $\left(\mathrm{F}_{3,50}=1.85, \mathrm{p}=0.150\right)$ et le charme $\left(F_{3.50}=5.68, p=0.002\right)$, sont plus abondantes dans les panses au printemps et en été (Tableau 3). Enfin, la mûre $\left(\mathrm{F}_{3.50}=6.83, \mathrm{p}=0.001\right)$ n'apparaît qu'à une seule saison (été) mais de façon très importante. 
TTABLEAU 3. - Abondance des diverses items alimentaires identifiés dans les panses en fonction de la saison (pourcentage moyen, par panse, du poids sec des divers items).

\begin{tabular}{|c|c|c|c|c|}
\hline Item alimentaire & Printemps & Eté & Automne & Hiver \\
\hline Anémone sylvie (Anemone nemorosa) & 5.2 & 0.0 & 0.0 & 0.0 \\
\hline Aubépine (Crataegus monogyna) & 4.5 & 0.1 & 0.0 & 0.0 \\
\hline Bouleau (Betula verrucosa) & 0.1 & 0.0 & 0.1 & 0.0 \\
\hline Bruyères (Calluma vulgaris, Lirica cincrea) & 0.0 & 0.0 & 0.9 & 0.4 \\
\hline Champignons & 0.2 & 0.7 & 2.4 & 3.2 \\
\hline Charme (Carpimus betulus) & 10.4 & 4.6 & 0.0 & 0.0 \\
\hline Chênes (Quercus spp.) & 1.9 & 4.1 & 0.3 & 0.0 \\
\hline Chèvrefeuille (Lonicera peryclimenum) & 12.7 & 14.6 & 4.1 & 2.4 \\
\hline Germandréc (Teucrium scorodonia) & 0.1 & 0.1 & 0.1 & 0.7 \\
\hline Glands & 5.1 & 1.4 & 59.8 & 16.8 \\
\hline Graminées & 0.0 & 0.0 & 1.0 & 0.6 \\
\hline Houx (Ilex aquifolium) & 0.0 & 0.0 & 0.0 & 0.1 \\
\hline Labiées & 0.0 & 0.0 & 0.1 & 0.0 \\
\hline Lierre (Hedera helix) & 0.4 & 1.5 & 3.0 & 3.8 \\
\hline Ligneux & 28.7 & 17.3 & 10.1 & 12.3 \\
\hline Monocotylédones & 0.0 & 0.0 & 0.0 & 0.0 \\
\hline Mousses & 0.1 & 0.0 & 0.2 & 0.1 \\
\hline Mûres & 0.0 & 14.7 & 0.0 & 0.0 \\
\hline Onagracées & 0.0 & 0.0 & 0.0 & 0.0 \\
\hline Prunellier (Prunus spinosa) & 0.0 & 0.0 & 0.0 & 0.0 \\
\hline Ronce (Rubussp.) & 25.8 & 36.8 & 17.0 & 57.5 \\
\hline Troène (ligustrum vulgare) & 0.0 & 0.4 & 0.2 & 0.0 \\
\hline Animal & 0.1 & 0.0 & 0.1 & 0.1 \\
\hline Indéterminés & 4.7 & 3.5 & 0.7 & 2.2 \\
\hline TOTAL & 100.00 & 100.00 & 100.00 & 100.00 \\
\hline
\end{tabular}

La seconde partie de l'analyse a permis de mettre en évidence des différences significatives d'abondance des items végétaux dans les panses entre les sexes en été $\left(\mathrm{F}_{19.377}=1.89, \mathrm{p}=0.012\right)$ et en hiver $\left(\mathrm{F}_{19.503}=1.61, \mathrm{p}=0.046\right)$, mais ni au printemps $\left(F_{19,410}=0.28, p=0.999\right)$, ni en automne $\left(F_{59,629}=1.31, p=0.164\right)$. En été, les différences concernent la ronce dont les femelles ont tendance à plus consommer les 
feuilles $\left(\mathrm{F}_{1.16}=2.97, \mathrm{p}=0.104\right)$ alors que les mâles se tournent plutôt vers les fruits (mûres: $\mathrm{F}_{1.16}=1.40, \mathrm{p}=0.255$ ) (Fig. $2 \mathrm{a}$ ). En hiver, la ronce est encore en cause, les femelles tendant à en manger plus que les mâles $\left(F_{1,22}=1.62, \mathrm{p}=0.217\right)$ qui pour leur part continuent à consommer les glands $\left(F_{1.22}=1.34, p=0.259\right)$ tombés en automne, ainsi que des champignons $\left(F_{1.22}=2.56, p=0.124\right)$ (Fig. 2b).
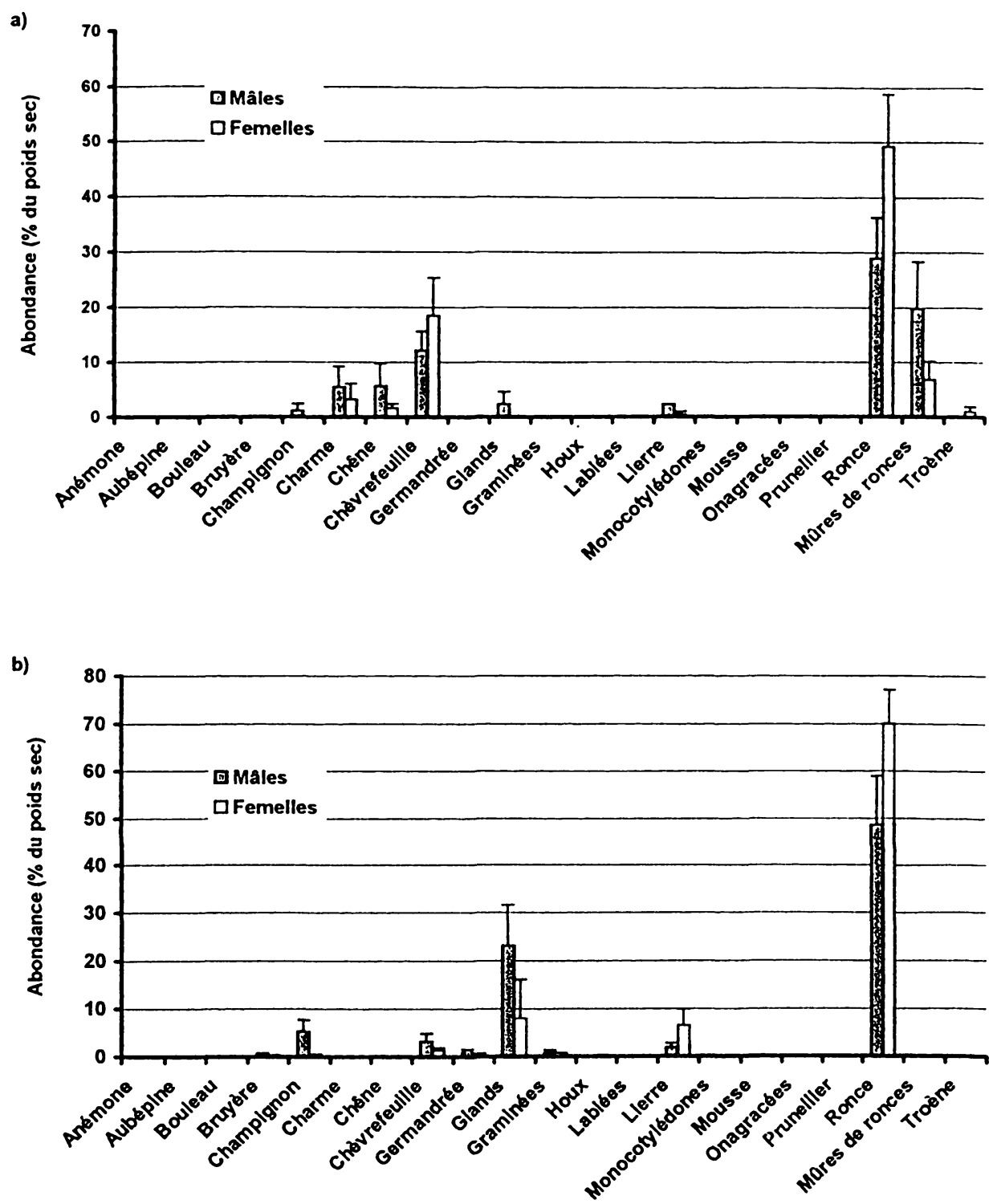

Fig. 2. - Abondance (\% du poids sec) des items alimentaires dans les panses de chevreuil des deux sexes : a. en été, b. en hiver. Les barres représentent l'erreur type. 


\section{DISCUSSION}

Bien qu'étant réalisée en période de forte densité, la disponibilité des différents items alimentaires sur le site d'étude est restée comparable à celle de faible densité (Ballon et al. 1992). De plus, les échantillons prélevés sont répartis sur l'ensemble de la forêt sans privilégier de zones particulières. Ces résultats semblent donc généralisables pour les chevreuils forestiers dans la limite de précision de la méthode d'analyse utilisée. Par exemple, la quantification de la consommation de certains items (comme les mûres et les champignons), par leur poids sec, peut sembler peu représentative.

Les items alimentaires trouvés dans les panses de chevreuils dans cette étude sont habitucllement cités comme composants principaux du régime alimentaire du chevreuil (Tixier et Duncan 1996). Ce sont notamment des feuilles d'arbres et d'arbustes et des graines/fruits. Cependant, dans notre étude, seuls la ronce et le chèvrefeuille sont présents dans la quasi-totalité des panses à chaque saison (Tableau 2). En comptant les six items les plus abondants (ronces, mûres, chèvrefeuille, charme, glands, ligneux non identifiés), nous prenons en compte entre 80 et $90 \%$ du poids sec du contenu des panses (Tableau 3). Ces résultats confirment l'observation qu'une grande partie du régime alimentaire du chevreuil est composée d'un nombre relativement peu élevé d'items consommés majoritairement (Duncan et al. 1998). D'après Tixier et al. (1997), le chevreuil consomme les espèces peu fibreuses en préférant celles riches en sucres solubles, mais également en tanins.

Nos résultats ont montré que, même si le chevreuil apparaît comme une espèce sélective, il est malgré tout fortement opportuniste dans sa façon d'exploiter la variabilité saisonnière d'abondance et de composition des végétaux (voir Tixier et al. 1997). En effet, mis à part la ronce et le chèvrefeuille, il montre des pratiques alimentaires tranchées entre l'automne/hiver d'une part et le printemps/été d'autre part. Dans le premier cas, les items consommés incluent le lierre, les champignons, les bruyères, les glands et même les graminées, dans l'autre cas, le chevreuil mange plutôt le charme, le chêne, l'anémone sylvie, I'aubépine et les mûres. De la même façon, Danilkin et Hewison (1996) notent que le rapport entre les herbacées non graminéennes et les arbres et arbustes dans le régime alimentaire du chevreuil passe de 20/80 en automne-hiver à $35 / 65$ et même à 75/25 en printemps-été.

Cette variabilité saisonnière est particulièrement marquée dans le cas des graines et des fruits. Uniquement disponibles ponctuellement, ils sont intensivement consommés pendant une certaine saison. Ainsi, les glands sont présents dans plus de $90 \%$ des panses et composent plus de la moitié du poids sec des panses en automne (voir aussi Maillard et Picard 1987). Cette ressource est donc très importante à une saison où le chevreuil est en train de récupérer sa condition corporelle (Hewison et al. 1996) suite aux activités reproductrices. Elle pourrait être également à l'origine de variations démographiques de la population selon sa disponibilité annuelle (Massei et al. 1996).

Nous n'avons répertorié que peu d'études qui se soient intéressées aux différences de régime alimentaire entre sexes chez le chevreuil. Dans celle de Cibien et al. (1995) sur le chevreuil de plaine, aucune différence de régime n'apparaît entre les sexes. Globalement, il y a peu de différences de contenu entre les panses des deux sexes dans notre échantillon de Dourdan (voir aussi Duncan et al. 1998), mais lorsqu'elles existent, ces différences concernent une part non négligeable du régime (25 à $40 \%$ ). Deux items seulement sont exploités de manière différente par les mâles et les femelles et représentent des ressources ponctuellement disponibles (Fig. 2). La forte abondance des mûres dans le régime du chevreuil en été ne concerne que les mâles, les femelles 
exploitant plus les feuilles de ronce que les fruits. Ceci pourrait s'expliquer par une recherche particulière de sucre chez les mâles, en rut à cette période (Strandgaard 1972). On pourrait y voir aussi la conséquence d'une différence d'utilisation de l'espace. Les mâles fréquentent des zones plus ouvertes pour leurs activités de patrouille, et les bordures du territoire suivant les chemins, les ruisseaux et d'autres zones de lisière en général pour leurs activités de marquage du territoire (Hewison et al. 1998). La ronce fructifie plus facilement dans ces zones où la lumière pénètre bien. La seconde différence de régime alimentaire entre les sexes provient de la poursuite de l'exploitation des glands par les mâles pendant l'hiver. Les femelles qui, à l'inverse, en mangent peu apres l'automne, continuent encore à consommer des ronces. Les mâles exploitent aussi les champignons à cette saison. On peut poser l'hypothèse que la consommation de glands chez le mâle correspond à la recherche d'éléments métaboliques particuliers et d'un apport énergétique important. Une hypothèse de type comportemental pourrait être proposée à l'exploitation des glands par les mâles en février. C'est en effet, à cette période que les mâles commencent à définir et à marquer leur territoire par grattis et frottis (Johansson et Liberg 1996). Il est donc possible que pendant ce marquage le brocard découvre les glands cachés sous la litière et continue de les exploiter de manière opportuniste.

Nous avons montré la dominance de la ronce et du chèvrefeuille dans le régime alimentaire du chevreuil à Dourdan. Par ailleurs, pendant la même période que les prélèvements de panses, une mesure de la pression d'abroutissement par le chevreuil sur les espèces lignifiées a été effectuée (Ballon et al. 1992). L'inventaire de la pression de consommation exercée par les Cervidés sur la flore lignifiée a été réalisé juste avant les débourrements de la végétation. Il relève les traces de consommation des Cervidés depuis l'arrêt de la végétation jusqu'à son débourrement. Cet inventaire permet de cálculer la fréquence de consommation des espèces lignifiées les plus fréquentes, en calculant le rapport du nombre de placettes avec au moins $5 \%$ de consommation sur le nombre de placettes avec au moins une présence, ceci pour chacune des espèces végétales lignifiées (sauf le lierre). Parmi les espèces concernées, seule la ronce $(69,4 \%) \mathrm{et}$, à un moindre degré, le chèvrefeuille $(21,4 \%)$ ont montré une pression d'abroutissement élevée. Nos résultats sont donc en accord avec ces estimations de l'impact du chevreuil sur les différentes espèces et confortent l'utilisation du niveau d'abroutissement pour la gestion de l'équilibre entre la population de chevreuils et son milieu (Guibert 1997).

\section{REMERCIEMENTS}

Ce travail a été réalisé grâce à la collaboration technique et financière de l'Office national des Forĉts. Nous remercions P. Duncan, H. Verhcyden et un rapporteur anonymc pour leurs commentaires sur le manuscrit.

\section{BIBLIOGRAPHIE}

Andersen, R, J.-M. Gaillard, O. Liberg ct C. SAN Jose, 1998. - Variation in life-history parameters. Pp. 285-307, in: The European roe deer: the biology of success. Eds. Andersen et al., Scandinavian University Press, Oslo. 
Badia, J., M. Goulard, J. Rech et L. Faliu, 1986. - Guide d'utilisation. Plantox, système d'identification des plantes toxiques (version 86.1).I.N.R.A.-E.N.V.T., Toulouse.

Ballon, P., B. Guibert, J.-P. Hamard et Y. Boscardin, 1992. - Evolution of roe deer brousing pressure in the forest of Dourdan. Pp. 513-515, in: Ongulés/Ungulates 91. Eds Spitz et al., S.F.E.P.M.-I.R.G.M., Toulouse.

Bideau, E., J.-P. Vincent et J.-M. Angibault, 1983. - Occupation de l'espace chez le chevreuil (Capreolus capreolus), 1. - Cas des mâles. Acta Oecologica, Oecologia Applicata, 4: 163-184.

Boisaubert, B. et D. Mouron, 1997. - La situation du chevreuil en France. Bulletin mensuel de l'O.N.C., 218: 22-25.

Cederlund, G., H. Luungouist, G. Markgren et F. Stalfelt, 1980. - Foods of moose and roc deer at Gimsö in central Sweden. Results of rumen contents analysis. Viltrevy, 11: 169247.

Cibien, C., E. Bidenu, B. Boisaubert, H. Biran et J.-M. Angibault, 1995. - Seasonal diet and habitat use in field roe decr (Capreolus capreolus) in the Picardie region. Gibier Faune Sauvage, Game Wildlife, 12: 37-49.

Cornelis, J., J. CASAer et M. Hermy, 1999. - Impact of season, habitat and research techniques on diet composition of roc deer (Capreolus capreolus): a review. J. Zool., Lond., 248: 195-207.

Crawiey, M.J., 1993. - GLIM for ecologists. Blackwell Scientific, Oxford.

Danilkin, A. et A.J.M. Hewison, 1996. - Behavioural ecology of Siberian and European roe deer. Chapman et Hall, London.

Duncan, P., H. Tixier, R.R Hofmann, et M. LeChiner-Doll, 1998. - Fceding strategies and the physiology of digestion in roc decr. Pp. 91-116, in: The European roe deer: the biology of success. Eds. Andersen et al., Scandinavian University Press, Oslo.

Groupe Cievreuil françAis, 1999. - Suivi des populations de chevreuils. Actes du colloque de Lyon, 26-27 novembre. 1998. Bulletin mensuel de l'O.N.C., 244: 140 pp.

GUIBERT, B., 1997. - Une nouvelle approche des populations de chevreuils en forêt; l'indice de pression sur la flore. O.N.F.: Bulletin Technique, 32: 5-13.

Hewison, A.J.M., J.-P. Vincent et D. Reby, 1998. - Social organisation of European roe deer Pp. 189-219, in: The European roe deer: the biology of success. Eds. Andersen et al. University Press, Oslo.

Hewison, A.J.M., J.-M. Angibault, J. Boutin, E. Bideau, J.-P. Vincent et A. Sempere, 1996. Annual variation in body composition of roe deer (Capreolus capreolus) in moderate environmental conditions. Canadian Journal of Zoology, 74 :245-253.

Hewison, A.J.M., J.-P. Vincent, J.-M. Angibault, D. Delorme, G. Van Laere et J.-M. GAILLARD, 1999. - Tests of age cstimation from tooth wear on roe deer of known age: variation within and between populations. Canadian Journal of Zoology, 77 :58-67.

JOHANSSON, A. ct O. LiBERG, 1996. - Functional aspects of marking behaviour by male roe deer (Capreolus capreolus). Journal of Mammalogy, 77: 558-567.

MAILl_ARD, D. et J.-F. PICARD, 1987. - Le régime alimentaire automnal et hivernal du chevreuil (Capreolus capreolus), dans une hêtraie calcicole, déterminé par l'analyse des contenus stomacaux. Gibier Faune Sauvage, 4: 1-30.

Maizeret, C., J.-M. Boutin, C. Cibien et J.-P. CarLino, 1989. - Effects of population on the dict of roc decr and the availability of their food in the Chizé forest. Acta Theriologica, $34: 235-246$

Massei, G., P.V. Genov. et B.W. Staines, 1996.- Diet, food availability and reproduction of wild boar in a Mediterranean coastal area. Acta Theriologica, 41: 307-320.

Sempere, A.J., R Mauget et C. Mauget, 1998. - Reproductive physiology of roe deer. Pp. 161188, in: The European roe deer: the biology of success. Eds. Andersen et al., Scandinavian University Press, Oslo. 
STRANDGAARD, H., 1972. - An investigation of corpora lutea, embryonic development and tme of birth of roc deer (Capreolus capreolus) in Denmark. Danish Review of Gome Biology, 6: 1-22.

Tixiler, H. et P. Duncan, 1996. - Are European decr browsers ? A review of variations in the composition of their diets. Revue d'Ecologie (Terre et Vie), 51: 3- 17.

Tixier, H., P. Duncan, J. Scehovic, A. Yani, M. Gleizes et M. Lil.a, 1997. - Food selection by European roe deer (Capreolus capreolus): effects of plant chemistry, and consequenes or the nutritional value of their diets. Journal of Zoology, London, 242: 229-245.

VINCENT, J.-P., J.-M. GAIILARD et E. Bidiau, 1991. - Kilometric index as biological indicitor for monitoring forest roe deer populations. Acta Theriologica, 36: 315-328.

Vinclent J.-P., E. Bibeau, A.J.M. Hizison ct J.-M. ANGibault, 1995. - The influence of increasing density on body weight, kid production, home range size and winter grouping in roc decr. Journal of Zoology, London, 236: 371-382. 